\title{
Two-point motional Stark effect diagnostic for Madison Symmetric Torus ${ }^{a)}$
}

\author{
J. Ko, ${ }^{1, b)}$ D. J. Den Hartog, ${ }^{1}$ K. J. Caspary, ${ }^{1}$ E. A. Den Hartog, ${ }^{1}$ N. A. Pablant, ${ }^{2}$ \\ and H. P. Summers ${ }^{3}$ \\ ${ }^{1}$ University of Wisconsin-Madison, Madison, Wisconsin 53706, USA \\ ${ }^{2}$ University of California-San Diego, La Jolla, California 92093, USA \\ ${ }^{3}$ University of Strathclyde, Glasgow G1 1XQ, Scotland, United Kingdom
}

(Presented 18 May 2010; received 17 May 2010; accepted 28 May 2010; published online 1 October 2010)

\begin{abstract}
A high-precision spectral motional Stark effect (MSE) diagnostic provides internal magnetic field measurements for Madison Symmetric Torus (MST) plasmas. Currently, MST uses two spatial views - on the magnetic axis and on the midminor (off-axis) radius, the latter added recently. A new analysis scheme has been developed to infer both the pitch angle and the magnitude of the magnetic field from MSE spectra. Systematic errors are reduced by using atomic data from atomic data and analysis structure in the fit. Reconstructed current density and safety factor profiles are more strongly and globally constrained with the addition of the off-axis radius measurement than with the on-axis one only. () 2010 American Institute of Physics. [doi:10.1063/1.3461995]
\end{abstract}

\section{INTRODUCTION}

Motional Stark effect (MSE) diagnostic ${ }^{1}$ provides internal magnetic field measurements in magnetically confined toroidal plasmas by analyzing polarized atomic emissions due to Lorentz electric field $(E)$. Due to the low magnetic fields $(\leq 0.5 \mathrm{~T})$ in Madison Symmetric Torus (MST) reversed-field pinch plasmas, ${ }^{2}$ it is not practical to select a particular Stark component in the signal. This precludes using the conventional method to infer the direction of the polarization, relying on photoelectric modulator polarimetry technique. Instead, MST has adopted spectroscopic method based on the atomic emission models. ${ }^{3,4}$ Unlike tokamaks, the magnitude of the (toroidal) magnetic field on the magnetic axis $(|B|)$ is unknown since the toroidal field in this region is largely generated by poloidal current flowing in the edge, not by external toroidal field coils. Therefore, $|B|$ at the magnetic axis is an important constraint in reconstructing the reverses field pinch (RFP) equilibria. Recently, MST has installed one more spatial view off the magnetic axis by half its minor radius ("off-axis view") in addition to the view on the magnetic axis ("on-axis view") to better constrain magnetic equilibrium reconstructions. Figure 1 shows the geometry of these two systems. The radial resolutions are 5 (off-axis) and 6 (on-axis) $\mathrm{cm}$ with the diagnostic neutral beam (DNB) with $45-50 \mathrm{keV}$ and $5 \mathrm{~A}$ and the time resolution is about $0.1-0.5 \mathrm{~ms}$. Unlike the on-axis view, both $|B|$ and its direction are unknown at the off-axis view and the analysis should provide both pieces of information from the MSE spectra. Since $E$ is almost vertical near the magnetic axis, a vertical polarizer suppresses almost all $\sigma$ components of the Stark multiplets from the on-axis spectra. Since the pitch angle is also unknown at the off-axis view, this kind of "weighted"

\footnotetext{
a) Contributed paper, published as part of the Proceedings of the 18th Topical Conference on High-Temperature Plasma Diagnostics, Wildwood, New Jersey, May 2010.

${ }^{b)}$ Electronic mail: jko6@wisc.edu.
}

selection is not available at the off-axis view. Instead, the off-axis view uses two separate optical paths each of which includes a polarizer. The transmission axes of these two polarizers are perpendicular to each other. The analysis scheme developed for the off-axis (and also on-axis, in general) spectra is introduced in Sec. II where the application of atomic data and analysis structure (ADAS) calculations to the spectrum fit is included. Section III presents the study of sensitivity in the magnetic equilibrium reconstructions to the twopoint MSE constraints and the conclusions are given in Sec. IV.

\section{ANALYSIS SCHEME}

Voslamber ${ }^{5}$ derived the Stokes vectors $S^{\sigma}$ and $S^{\pi}$ for $\sigma$ and $\pi$ radiations, respectively, in an arbitrary geometry as follows:

$$
\begin{aligned}
& S^{\sigma}=\left[\begin{array}{l}
I_{0}^{\sigma} \\
I_{1}^{\sigma} \\
I_{2}^{\sigma} \\
I_{3}^{\sigma}
\end{array}\right]=\left[\begin{array}{c}
I^{(\sigma) n p}+I_{\perp}^{\sigma}\left(1+\sin ^{2} \theta \sin ^{2} \phi\right) \\
I_{\perp}^{\sigma}\left(\cos ^{2} \phi-\cos ^{2} \theta \sin ^{2} \phi\right) \\
I_{\perp}^{\sigma} \cos \theta \sin 2 \phi \\
0
\end{array}\right], \\
& S^{\pi}=\left[\begin{array}{c}
I_{0}^{\pi} \\
I_{1}^{\pi} \\
I_{2}^{\pi} \\
I_{3}^{\pi}
\end{array}\right]=\left[\begin{array}{c}
I^{(\pi) n p}+I_{\perp}^{\pi}\left(1-\sin ^{2} \theta \sin ^{2} \phi\right) \\
-I_{\perp}^{\pi}\left(\cos ^{2} \phi-\cos ^{2} \theta \sin ^{2} \phi\right) \\
-I_{\perp}^{\pi} \cos \theta \sin 2 \phi \\
0
\end{array}\right],
\end{aligned}
$$

where $I^{(\sigma) n p}$ and $I^{(\pi) n p}$ are the intensities of the unpolarized background for each $\sigma$ and $\pi$ radiations, respectively, and $I^{\sigma}{ }_{\perp}$ and $I^{\pi}{ }_{\perp}$ are the intensities of pure $\sigma$ and $\pi$ radiations, respectively, in directions perpendicular to $E$. The coordinate system for this derivation is explicitly given in Fig. 1(a) in Voslamber. ${ }^{5}$ The neutral beam direction and the line of sight constitute the $x-z$ plan where $z$ axis represents the beam direction and $\theta$ indicates the viewing angle subtended from the $z$ axis. $\phi$ is the angle on the $x-y$ plane subtended from 

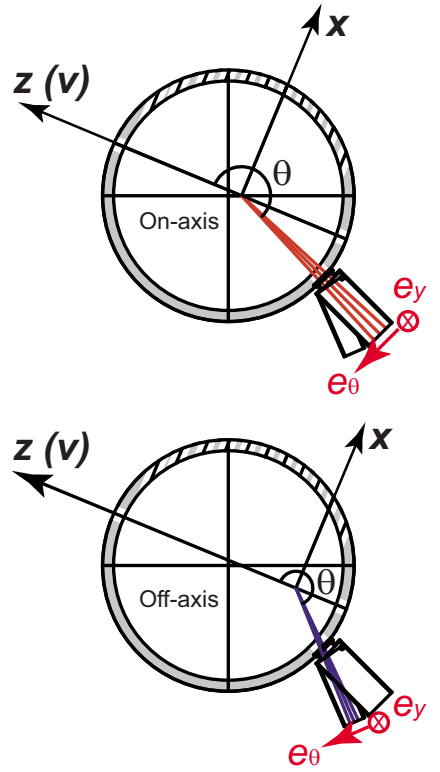

FIG. 1. (Color online) MST geometry in the coordinate system given in Fig. 1(a) in Voslamber (Ref. 5) for the on-axis (top) and off-axis (bottom) MSE views. The polarizer frame of reference is also given in both views $\left(\mathbf{e}_{\theta}\right.$ and $\mathbf{e}_{y}$ ). The small rectangles at the lower right of the figures indicate the position and shape of the periscopes.

the $x$ axis to the $B_{\perp}$, the projection of the magnetic field $B$ on the $x-y$ plane. The polarizer frame of reference can be constructed such that the increasing $\theta$ direction is its horizontal axis $\left(\mathbf{e}_{\theta}\right)$ and the $y$ direction is its vertical axis $\left(\mathbf{e}_{y}\right)$, resulting in its transmission axis, $\alpha$, as shown in Fig. 1(a) in Voslamber. ${ }^{5}$ The measured intensity after the polarizer can be obtained from the first Stokes parameter from the Muller matrix ${ }^{6}$ calculation for the system with a polarizer for both $\sigma$ and $\pi$ radiations. The first parameter of the resultant Stokes vector is

$$
S_{p}(0)=\frac{1}{2}\left[I_{0}+I_{1} \cos (2 \alpha)+I_{2} \sin (2 \alpha)\right],
$$

where $I_{i}$ are from Eq. (1) for $\sigma$ radiation and Eq. (2) for $\pi$ radiation.

Equation (3) with the appropriate geometric parameters for MST can be used to analyze MSE spectra. Figure 1 illustrates the MST geometry in the coordinate system given in Fig. 1(a) in Voslamber. ${ }^{5}$ By virtue of the circular nature in the plasma cross section in MST, the magnetic field vector always lies on the $x-y$ plane, i.e., $B=B_{\perp}$. The spectrum fit is performed to determine the pitch angle $\left(90^{\circ}-\phi\right)$, the spacing between the Stark multiplets $(|B|)$, and the intensity scaling factor. The relative intensities among the Stark multiplets $\left(I_{\perp}\right.$ 's) are obtained (and fixed during the fit) from either $\mathrm{Mandl}^{7}$ or ADAS package. ${ }^{8}$ Figure 2 shows an example of the fit for both on- and off-axis spectra where the raw spectra overplotted with the $\sigma, \pi$, and total Gaussian curves. From these fits, $|B|$ is $0.56 \pm 0.02 \mathrm{~T}$ from the on-axis, and $0.47 \pm 0.08 \mathrm{~T}$ from the off-axis. The pitch angles are effectively zero at the on-axis and $40.02 \pm 9.9^{\circ}$ at the off-axis.

The fundamental drawback here is then the fact that it uses the theoretical and fixed $I_{\perp}$. For example, $I_{\perp}$ values from $\mathrm{Mandl}^{7}$ are those calculated with the electric field $=10 \mathrm{MV} / \mathrm{m}$. The approximate Stark electric field in MST
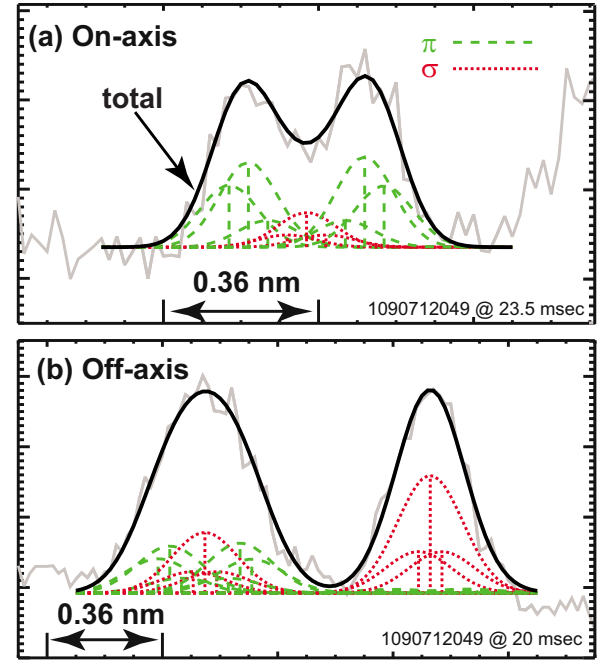

FIG. 2. (Color online) An example of the MSE spectrum fit from (a) on-axis and (b) off-axis system. The raw spectra (gray) are overplotted with $\sigma$ (dotted), $\pi$ (dashed), and total (solid) Gaussian fit curves. Note that there are two sets of Stark multiplets from the off-axis view which has two separate optical paths.

with the $45 \mathrm{keV}$ beam and $B \approx 0.5 \mathrm{~T}$ is about $1.5 \mathrm{MV} / \mathrm{m}$ which is an order of magnitude smaller. Besides, the simple linear Stark effect, which is assumed in this analysis, can be comparable with the Zeeman effect and fine structures due to spin-orbit coupling. The sensitivity in $|B|$ on the Stark multiplets' relative intensities has been investigated by fitting the on-axis spectra with different sets of relative intensities obtained from ADAS which takes into account these effects. Figure 3 shows the on-axis $|B|$ from the MSE spectrum fit as a function of Lorentz electric field with which ADAS can produce different sets of relative intensities. One can calculate a new Lorentz electric field from the beam energy and $|B|$ from the spectrum fit and can compare it with that which corresponds to the set of relative intensities used in the fit. Only one $|B|$ value can have the identical Lorentz fields and this $|B|$ is used to normalize the other magnetic fields in Fig. 3. This result implies errors less than $2 \%$ in the inferred $|B|$ due to the uncertainties in the relative intensities can exist. This is far less than $20 \%-40 \%$ change in the intensities

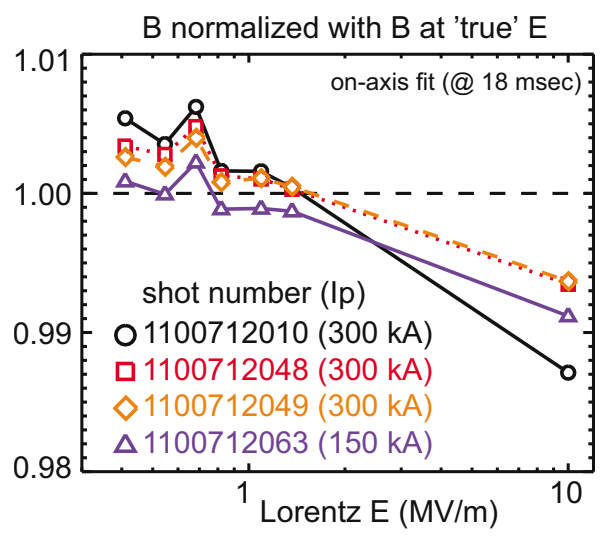

FIG. 3. (Color online) $|B|$ obtained from the MSE spectrum fit using several sets of relative intensities among Stark multiplets as a function of Lorentz fields. The data are normalized by $|B|$ at "true" Lorentz field. 

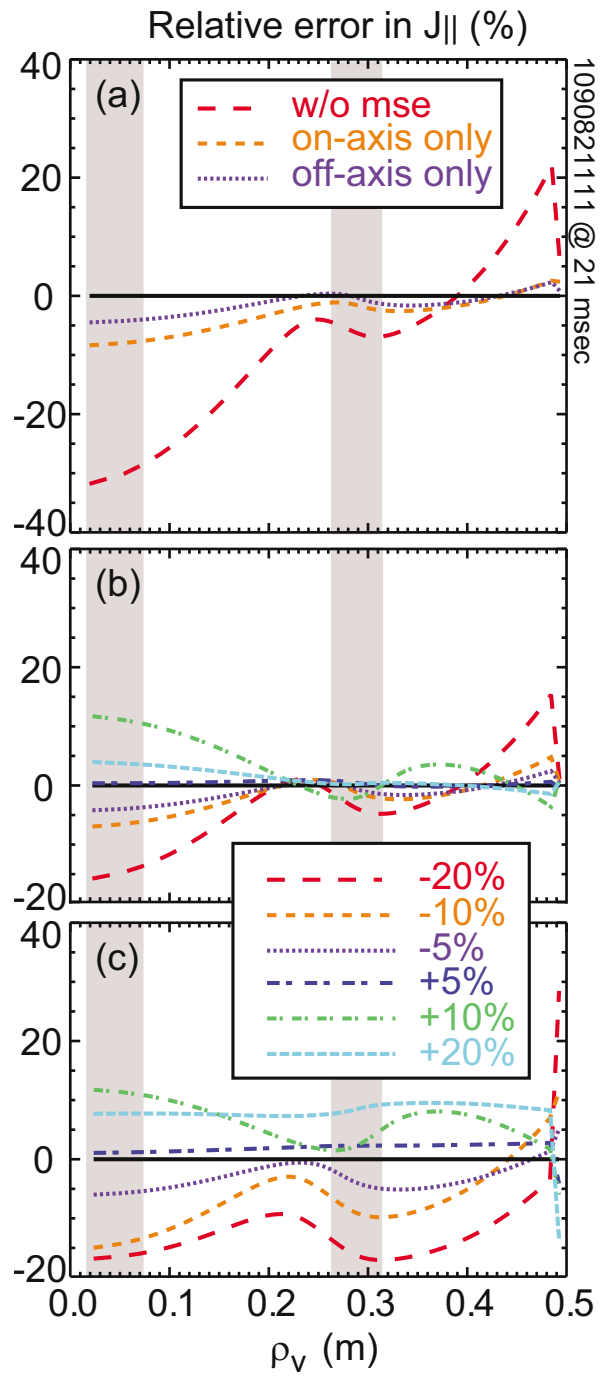

FIG. 4. (Color online) Fractional error (\%) in $J_{\|}$profiles from MSTFIT using MSE data with some artificial offsets. The shaded areas indicate the MSE finite radial resolutions. $\rho_{v}$ is the effective minor radius defined by the volume of flux surface.

themselves over the same range of the Lorentz field. This is also less than the typical statistical uncertainties in inferred $|B|$, which is about $2 \%-4 \%$.

\section{MSTFIT SENSITIVITY TO MSE}

One of the very important usages of MSE data is to constrain magnetic equilibrium reconstructions. MST has a nonlinear, axisymmetric, fixed boundary Grad Shafranov solver, called MSTFIT, ${ }^{9}$ to fit its own RFP equilibria (analogous to EFIT etc. in the tokamak community). Parallel current density $\left(J_{\|}\right)$is calculated using MSTFIT with a range of onand off-axis MSE measurements which deviate by up to $\pm 20 \%$ from the measured values in a nominal RFP discharge. Three cases have been tested: (a) The effect with and without the MSE constraints are tested; (b) the off-axis MSE values are fixed with the measured values and the on-axis MSE values vary about the measured values by $\pm 20 \%$; and (c) the on-axis MSE values are fixed with the measured values and the off-axis MSE values vary about the measured values by $\pm 20 \%$. Cases (b) and (c) mainly examine the error propagation to the equilibrium profiles from on- and off-axis MSE data, respectively. Figures 4(a)-4(c) show the corresponding cases mentioned above. Each shows the errors in $J_{\|}$ profiles relative to the fully constrained profile. From Fig. 4(a), it is obvious that the deviation in the profiles is huge without any internal magnetic constraints. It also shows that the off-axis data constrain the profile better than the on-axis data can. Figures 4(b) and 4(c) imply that the off-axis MSE errors propagate the whole profile whereas the effect of the on-axis errors is mainly local. The relative errors in the profiles are roughly linear to the errors in the off-axis MSE data.

\section{CONCLUSIONS}

The Stokes vector approach with correct geometry treatment gives the analysis scheme to provide both the direction and magnitude of internal magnetic fields. Statistical uncertainties in this analysis are about $2 \%-4 \%$ with the DNB from FY2009. This is larger than the systematic uncertainty due to the usage of fixed relative intensities for Stark multiplets, which is much less than $2 \%$. The off-axis MSE data constrain RFP equilibria much more strongly and globally than the on-axis data, the relative errors in the profiles being roughly linear to the error in the off-axis data.

\section{ACKNOWLEDGMENTS}

This work was supported by the U.S. Department of Energy under Contract No. DE-FC02-05ER54814.

${ }^{1}$ F. M. Levinton, R. J. Fonck, G. M. Gammel, R. Kaita, H. W. Kugel, E. T. Powell, and D. W. Roberts, Phys. Rev. Lett. 63, 2060 (1989).

${ }^{2}$ R. N. Dexter, D. W. Kerst, T. W. Lovell, and S. C. Prager, Fusion Technol. 19, 131 (1991).

${ }^{3}$ D. Craig, D. J. Den Hartog, and G. Fiksel, Rev. Sci. Instrum. 72, 1008 (2001).

${ }^{4}$ D. J. Den Hartog, D. Craig, D. A. Ennis, G. Fiksel, S. Gangadhara, D. J. Holly, J. C. Reardon, V. I. Davydenko, A. A. Ivanov, A. A. Lizunov, M.

G. O'Mullane, and H. P. Summers, Rev. Sci. Instrum. 77, 10F122 (2006).

${ }^{5}$ D. Voslamber, Rev. Sci. Instrum. 66, 2892 (1995).

${ }^{6}$ E. Hecht, Optics, 4th ed. (Addison-Wesley, Reading, 2002).

${ }^{7}$ W. Mandl, R. C. Wolf, M. G. V. Hellermann, and H. P. Summers, Plasma Phys. Controlled Fusion 35, 1373 (1993).

${ }^{8}$ H. P. Summers and L. Wood, "Atomic data and analysis structure," JET Report No. JET-IR(94)06, 1994.

9 J. K. Anderson, C. B. Forest, T. M. Biewer, J. S. Sarff, and J. C. Wright, Nucl. Fusion 44, 162 (2004). 